\title{
Research on Competency Evaluation for Standardization Professionals
}

\author{
Zhang Jingjuan ${ }^{1, *}$ Zhang Xinliang $^{1}$ Yu Fan $^{1}$ \\ ${ }^{1}$ China National Institute of Standardization, Beijing 100191, China \\ ${ }^{*}$ Corresponding author. Email: zhangjingjuan@cnis.ac.cn
}

\begin{abstract}
Standardization is a professional technology, standardization professionals should have corresponding competency. Both standardization professionals and employers have actual demands for competency evaluation. This research surveyed and analysed these demands, as well as standardization evaluation activities and standardization literatures. Based on the results of survey and analysis, this research proposed the communal knowledge and skill system, and a competency evaluation framework for standardization professionals. At last, follow-up work suggestions and advice were given.
\end{abstract}

Keywords: standardization, professionals, competency, evaluation

\section{Introduction}

\subsection{Concepts Definition and Research Scope}

In this research, standardization professionals refer to those engaged in one or more of the following activities: development of standards, standardization management, standardization research, standardization services.

Competency means possession of sufficient knowledge or skill(Merriam-Webster).

Competency of standardization professionals includes the communal knowledge and skills about standardization, which should be applicable to training and evaluation. The specialized knowledge and skills in a certain field are not included in this research.

\subsection{Background}

As China is extensively practicing standardization reform, association standards adaptable to market diversification and enterprise standards highlighting technical advancement will experience allround development [1]. It means that China needs more and more standardization professionals in the future. Standardization is a kind of basic work [2]. The competency of standardization professionals directly affects the quality and level of standardization, which needs to be stimulated and cultivated, but also needs scientific and reasonable evaluation. Therefore, the research on competency evaluation for standardization professionals has practical significance.

In China, standardization training activities are organized by all standardization management departments at national, local and industrial level as well as standardization scientific research institutions and standardization associations. These activities make a certain contribution to the improvement of standardization professionals competency in China, but they are far from enough to meet the demand for standardization professionals training.

\subsection{Purpose}

Competency would be better able to predict important behaviors than would more traditional tests[3]. The purpose of this research is to complete a competency evaluation framework for standardization professionals, to provide operational technical support for standardization administration departments at all levels, enterprises and institutions to build standardization talents pool. At the sametime, it is helpful to make a career path plan for standardization professionals, attract and retain excellent talents to the standardization team.

\section{Methods}

This research was divided into three phases:

Phase one, demand survey. To obtain standardization professionals demand for training and enterprises demand for standardization professionals competency.

Phase two, current situation and literature survey. Analysis of the evaluation activities relevant to standardization, as well as the published monographs and textbooks on standardization.

Phase three, development and refinement of the competency evaluation framework. Designed the framework, and improved it through Delphi method.

\subsection{Phase one, demand survey}

This research analyzes the demand for standardization professionals competency from the perspectives of standardization professionals and enterprises. Training is an 
important way to acquire knowledge about standardization. Through the investigation of training on demand, we can analyze standardization professionals demand for knowledge. Through the enterprise recruitment information analysis, we can analyze enterprise demand for standardization professionals competency.

\subsubsection{Standardization professionals demand}

In this research, questionnaire survey was carried out for standardization professionals regarding training frequency, form, content, effect and other aspects. The statistical result of 392 valid questionnaires indicated that nearly $15 \%$ of respondents received standardization training every two years, while about $21 \%$ of respondents received standardization training for more than two years, and all respondents believed that they should receive standardization training at least once a year. The training of respondents mainly took the forms of short-term advanced study, relevant training session and in-house training. The respondents considered that the training content was not systematic and more than $50 \%$ of them concluded that there was only a modest effect of training.

According to the respondents, the standardization theory was most needed in their practical work of standardization, followed by professional knowledge related to standard development, field standardization, enterprise standard system, standardization frontier, international standardization and other aspects. See Figure 1 for the statistical result.

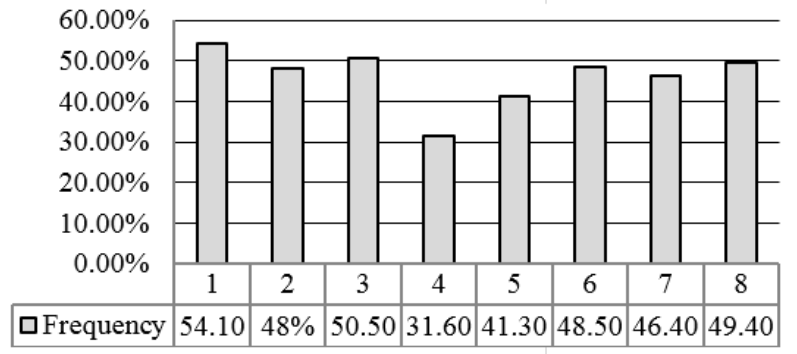

Figure 1 Standardization professionals demand for training content

The number in Figure 1 as below:

1 Standardization theory

2 Standardization frontier

3 Standard development

4 Standardization professional English

5 Standardization of laws and regulations

6 Enterprise standard system

7 International standardization

8 Field standardization

\subsubsection{Enterprises demand}

According to the requirements of the 480 standardization professional recruitment information, this research adopted qualitative approach to analyze the enterprises demand for standardization professionals competency. On the basis of vocabulary entry frequency and separately extract nine professional knowledges and skills including organization and coordination ability, communication skill, execution capability, etc. See Figure 2 for the statistical result. Enterprises demand for standardization professionals competency also reflects their demand for standardization training.

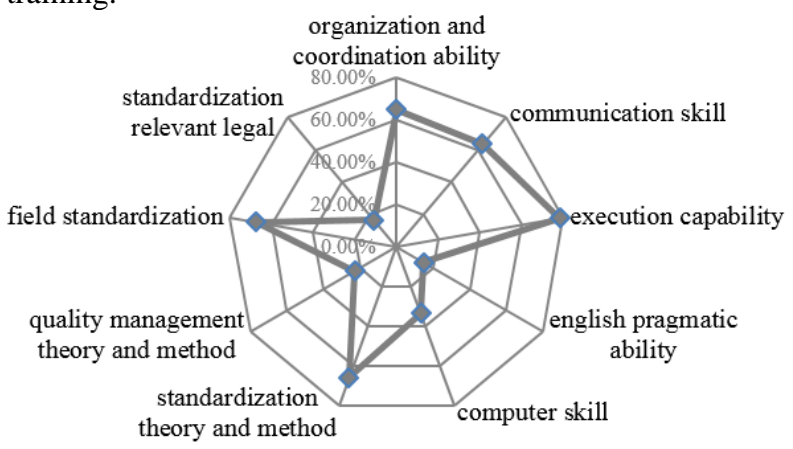

Figure 2 Enterprises demand for standardization professionals competency

\subsection{Phase two, present situation investigation}

This research did two things at this stage, one was to investigate the qualification exams for standardization professionals, the other was to collecte and analyze literatures about standardization. Through the investigation of relevant exams, this research found the problems existing in the current exams, and understood the social demand for competency evaluation for standardization professionals.

\subsubsection{Standardization professionals qualification exams}

Since 2005, Shanghai, Chongqing, Shenzhen, Nanjing, Anhui Province and other provinces and cities have carried out standardization professionals qualification exams successively. According to the investigation on such exams, this research concludes that the society has a strong demand for standardization professionals qualification exams, but current exams cannot meet the actual social demands for the following reasons:

Firstly, the professionals qualification exams are only applicable within limited scope, not covering all standardization practitioners. The standardization professionals qualification exams carried out in the provinces and cities mentioned above are basically limited to engineers, but the actual standardization has expanded beyond the field of engineering technology at the early stage to service industry, social management and other fields. The practitioners engaging in standardization of service industry, social management and other fields are excluded from current standardization engineer qualification exams.

Secondly, inconsistence in requirements of professionals qualification exams causes failure in trans-regional mutual 
recognition of qualifications and barrier to trans-regional flow of standardization professionals. Those provinces and cities carry out standardization engineer professional qualification exams by means of uniform organization, uniform outline, uniform exam paper and uniform criterion, but only by their own criteria. Therefore, even the qualification certificates at the same level are incomparable, causing failure in mutual recognition of qualifications and barrier to the trans-regional flow of standardization professionals.

\subsubsection{Monographs and textbooks on standardization}

The monographs and textbooks on standardization that had been published, were the summary of many years of work experience of standardization practitioners, or the concise results of standardization scientific research projects, or the crystallization of experts and scholars thinking on standardization knowledge. Therefore, the monographs and textbooks on standardization were the important basis and reference for this research to build the standardization professionals knowledge system and extract knowledge points.

Consideration on the objectivity of standardization development, the inheritance and accessibility of standardization knowledge, this research established the selection principles of monographs and textbooks: published after 2000; involved the basic and comprehensive knowledge on standardization; selected the latest version of similar content from the same author. Based on the above principles, this research extracted 31 monographs or textbooks. Through semantic analysis, this research extracted communal standardization knowledge from these literatures.

\subsection{Phase three, development and refinement of the competency evaluation framework}

On the basis of the prophase work, this research propose the knowledge system and skill system for standardization professionals, and present a competency evaluation framework, then Delphi method was used to verify and improve above results. 10 experts were recruited to a panelist, including 5 standardization experts, 5 human resources experts, questionnaires were sent to the panelist. After three Delphi iterations, a high consensus among experts was achieved.

\section{RESULTS}

\subsection{Knowledge system}

This research concludes that the communal knowledge of standardization professionals should include basic knowledge, core knowledge, relevant knowledge and frontier knowledge(see Figure 3).

Basic knowledge, including conceptual and factual knowledge to understand what the standardization refers to, is the entry-level knowledge for standardization professionals. Conceptual knowledge refers to basic concepts of standardization, while factual knowledge is present and historical knowledge on domestic and international standardization routine activities.

Core knowledge, including fundamental knowledge and practical knowledge, that is know-why and know-how, is the key knowledge to engage in standardization. Fundamental knowledge covers basic principle, basic method and mechanism of action of standardization, while practical knowledge includes development, implementation and supervision of standard, conformity assessment, basic general standards and practical English of international standardization.

Relevant knowledge mainly refers to supplementary knowledge to facilitate standardization professionals, including managerial knowledge, legal knowledge and economic knowledge. Managerial knowledge covers strategic management and project management; legal knowledge covers the laws and regulations related to standardization activities; economic knowledge refers to technological economics knowledge.

Frontier knowledge is the knowledge about the latest development and change of standardization, including international and national frontier practice and theory. Frontier theory shows the latest trend of standardization theory, technology and method, while frontier practice indicates the latest development of international and national standardization strategy and policy.

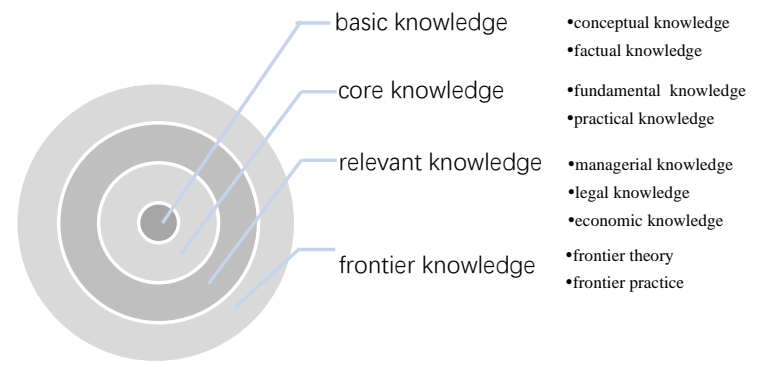

Figure 3 Communal knowledge of standardization professionals

\subsection{Skill system}

This research divides the communal skills of standardization professionals into three levels, that is, "primary", "intermediate" and "advanced".

Primary skill includes the ability to acquire and analyze standardization information and the ability to use dedicated standardization information tools.

Intermediate skill includes the ability to prepare normative standardization documents, the ability to distinguish the quality of normative standardization documents and the ability to organize and manage standardization projects. 
standardization professionals, irrespective of professionals background, provides a valuable guide for standardization management departments and standardization training institutions. It is helpful to form a uniform standardization professionals competency evaluation method and promote the regional mobility of standardization professionals.

\section{Suggestions and advice}

Standardization is a professional technology, standardization professionals should have corresponding competency. Competency needs to be clearly evaluated and normatively trained. Through competency evaluation, competency gaps could be identified, and then more customized training could be provided. Thus, a virtuous circle of standardization human resource construction can be formed, here are some Suggestions.

1)Build an education system. Refine and improve the knowledge system and skill system for standardization professionals. On this basis, research and develop education and training materials for standardization professionals, develop corresponding training courses. To train and establish a team of full-time and part-time teachers, and gradually establish an educational evaluation system that can train and select standardization professionals.

2)Establish unified evaluation standards. In order to promote construction of standardization talents pool, many local standardization administration departments have carried out the evaluation of standardization professionals. However, the scope and the scale of measurement are inconsistent. Therefore, a unified evaluation standard should be established to promote the construction of standardization talents pool.

3)Develop corresponding information system. Standardization professionals are scattered across industries and in different places, by using information technology and network platform, the development of standardization education and training courses can facilitate the independent learning of standardization professionals, break the boundaries of time and space, and effectively utilize education and training resources.

\section{REFERENCES}

[1] http://www.gov.cn/zhengce/content/201503/26/content_9557.htm

[2] Li chuntian. Urgent Matter of Standardization Talent Cultivation. J. World Standardization \& Quality Management. 1(2004) 5-6.

[3] McClelland D C. Testing for competence rather than for "intelligence".[J]. The American psychologist, 1973,28(1). 